\title{
Traditional and Complementary Medicine Use Among Adult Cancer Patients Undergoing Conventional Treatment in Sub-Saharan Africa: A Scoping Review on the Use, Safety and Risks
}

This article was published in the following Dove Press journal:

Cancer Management and Research

\author{
Amos Deogratius Mwaka (D) \\ Catherine Abbo $^{2}$ \\ Alison Annet Kinengyere $\mathbb{D}^{3}$ \\ 'Department of Medicine, School of \\ Medicine, College of Health Sciences, \\ Makerere University, Kampala, Uganda; \\ ${ }^{2}$ Department of Psychiatry, School of \\ Medicine, College of Health Sciences, \\ Makerere University, Kampala, Uganda; \\ ${ }^{3}$ Albert Cook Medical Library, College of \\ Health Sciences, Makerere University, \\ Kampala, Uganda
}

Background: Use of traditional and complementary medicine (T\&CM) is very common among patients in sub-Saharan Africa (SSA). However, there are limited data on concurrent use of T\&CM with conventional cancer therapies. In this scoping review, we sought to describe the (i) prevalence of use, (ii) types of medicine, (iii) reasons for taking T\&CM, (iv) current knowledge on safety and risks, (v) characteristics of adult cancer patients who use $\mathrm{T} \& \mathrm{CM}$, and (vi) perceived treatment outcomes among cancer patients undergoing conventional cancer treatment in SSA.

Methods: We conducted a systematic literature search for articles published in the English language in three scientific databases (PubMed, Embase and Web of Science). We used a scoping review approach to map relevant literature on T\&CM use among cancer patients undergoing conventional cancer treatments. We assessed 96 articles based on titles and abstracts, and 23 articles based on full text. Twelve articles fulfilled preset eligibility criteria.

Results: More than half of the included articles were from only two countries in SSA: Nigeria and Uganda. Median prevalence of use of T\&CM was 60.0\% (range: 14.1-79.0\%). Median percent disclosure of use of T\&CM to attending healthcare professionals was low at $32 \%$ (range: $15.3-$ $85.7 \%$ ). The most common reasons for non-disclosure were: the doctor did not ask, the doctor would rebuke them for using T\&CM, and the doctors do not know much about T\&CM and so there is no need to share the issue of use with them. T\&CM used by cancer patients included herbs, healing prayers and massage. Reported reasons for use of T\&CM in 8 of 12 articles included the wish to get rid of cancer symptoms, especially pain, cure cancer, improve physical and psychological well-being, treat toxicity of conventional cancer therapies and improve immunity. There were limited data on safety and risk profiles of T\&CM among cancer patients in SSA.

Conclusion: Use of traditional and complementary medicines is common among cancer patients undergoing conventional cancer treatments. Healthcare professionals caring for cancer patients ought to inquire and communicate effectively regarding the use of T\&CM in order to minimize the risks of side effects from concurrent use of T\&CM and biomedicines.

Keywords: traditional and complementary medicine, safety and risk profiles, cancer, conventional cancer therapy, sub-Saharan Africa

\section{Background}

In sub-Saharan Africa (SSA), traditional and complementary medicine (T\&CM) is widely used for various illnesses, including diabetes, cancer, hypertension and asthma. $^{1-5}$ A systematic review published in 2018 showed that patients use T\&CM
Correspondence: Amos Deogratius Mwaka

Email mwakaad@yahoo.com 
alone or in combination with conventional medicine, and that most of the T\&CM users (55.8-100\%) do not disclose use to the healthcare professionals, mainly for fear of retribution and because the healthcare professionals rarely ask patients about the use of T\&CM. ${ }^{1}$ T\&CM has been described by the World Health Organization (WHO) as

... the sum total of the knowledge, skills, and practices based on the theories, beliefs, and experiences traditional to different cultures, whether explicable or not, used in the maintenance of health as well as in the prevention, diagnosis, improvement or treatment of physical and mental illness.

and complementary medicine as

... a broad set of healthcare practices that are not part of that country's own tradition or conventional medicine and are not fully integrated into the dominant health-care system. $^{6,7}$

Generally, T\&CM can be described as any practice that is not part of conventional western medicine, whose philosophical underpinnings are beliefs, customs and experiences traditional to a particular group of people, and which is used in the maintenance of health, prevention of illnesses, determination of causes of ill-health, and treatment of diseases and ill-health, including physical, social and mental disorders. In spite of the encouragement by the WHO since 2000 that African nations and other low- and middle-income countries (LMICs) should embrace T\&CM, several nations in SSA have not incorporated T\&CM into their national health systems, mainly because of concerns over a lack of evidence on efficacy, effectiveness and risks of T\&CM. ${ }^{6}$ The Beijing Declaration of 2008 further emphasized the need for the LMICs and other nations to develop regulatory frameworks, promote research and incorporate $\mathrm{T} \& \mathrm{CM}$ into their national health systems in order to improve access to affordable healthcare to the populations. ${ }^{7,8}$ However, only a few nations in SSA, including the United Republic of Tanzania, South Africa, Sierra Leone, Ghana, Ethiopia and Cameroon, have thus far incorporated T\&CM into their national health systems and developed policies and regulations regarding their use. ${ }^{9-11}$ Inadequate understanding of T\&CMs used by patients clearly poses risks to their health. Similarly, inadequate knowledge by healthcare professionals on the mechanisms of action of T\&CMs and drug/herb interactions, because there are only a few medical schools that teach aspects of T\&CM, does not make things any better for the general population that uses T\&CM. Indeed, there is a low (19.6\%) level of knowledge of T\&CM among medical students and biomedical healthcare workers in Africa. ${ }^{12}$

The incidence of cancers has increased significantly worldwide, but more so in the LMICs, especially those in SSA. ${ }^{13-16}$ The majority of cancer patients in SSA are diagnosed in advanced stages, ${ }^{17-19}$ experience poor survival ${ }^{20-22}$ and often report the use of T\&CM, alone or in combination with conventional cancer therapies. For example, in Nigeria $34.5-65 \%$ of cancer patients used T\&CM, while in Ghana and Ethiopia the prevalence of use of T\&CM reached $73.5 \%$ and $79.0 \%$, respectively. ${ }^{3,23-25}$ In Uganda, $55-77 \%$ of cancer patients at the Uganda Cancer Institute used T\&CM concurrently with conventional cancer therapies. ${ }^{26,27}$ There are, however, limited data on the extent and trend in use of T\&CM among cancer patients undergoing conventional cancer therapies in most SSA countries. There are also limited data on the types of T\&CM used, and evidence of efficacy, effectiveness and risk associated with the use of each type of T\&CM commonly used by cancer patients in SSA. There are some data to show that several side effects have been reported with the use of T\&CM, including liver fibrosis, anemia, hyponatremia, bloody diarrhea and vaginal bleeding. ${ }^{28-31}$ It is generally difficult to know which patients have developed particular reactions as a result of T\&CM use because a large proportion of patients who use T\&CM rarely inform their physicians about concurrent T\&CM use..$^{27,32}$ The possibility of side effects and potential interactions between T\&CM and western medicines makes it imperative that primary healthcare providers gain interest, knowledge and positive attitudes towards T\&CM, and conduct well-designed scientific research to understand the pharmacodynamics and pharmacokinetics of T\&CM in order to determine their safety and risk profiles. There is evidence that the use of T\&CM is associated with interactions with conventional medicine, serious side effects and poor quality of life among cancer patients. ${ }^{33}$ However, this evidence is lacking for most T\&CMs commonly used by cancer patients in SSA. The majority of the few primary studies on T\&CM use among cancer patients in SSA had small sample sizes, included one of several cancers, and were heterogeneous in respect of the tools used in data collection as well as analysis approaches.

This scoping review sought to answer the following question: Among adult cancer patients undergoing conventional cancer treatment in sub-Saharan Africa, what is/are the (i) current extent of use of T\&CM, (ii) types of T\&CM in common use, (iii) reasons for using $\mathrm{T} \& \mathrm{CM}$, (iv) current 
knowledge on the safety and risks of use of T\&CM, (v) characteristics of the adult cancer patients who concurrently use $\mathrm{T} \& \mathrm{CM}$, and (vi) perceived treatment outcomes? The results of this synthesis of evidence on T\&CM use by cancer patients in SSA are expected to guide policy makers on the need for and process of integration of $\mathrm{T} \& \mathrm{CM}$ and conventional biomedical systems; inform the development and revision of medical school curricula in SSA countries; guide researchers on the appropriate investigations regarding $\mathrm{T} \& \mathrm{CM}$, including characterization of the users, drivers of use, types of T\&CM commonly used, patterns of use, efficacious and effective T\&CMs, side effects from direct use and from interaction of T\&CM with biomedical agents; and improve knowledge and attitudes of biomedical healthcare professionals towards the use of T\&CM by their patients.

\section{Methods}

\section{Study Design}

This is a scoping review aimed at generating knowledge on the current state of use, nature and extent of research conducted, and gaps in knowledge regarding the use of T\&CM among adult cancer patients in SSA. We conducted a scoping review because this approach to knowledge synthesis allows the inclusion of studies with different designs conducted over time to be comprehensively collated, summarized and interpreted to gain insights into the phenomenon under investigation, thereby informing interventions and research to improve outcomes. In particular, scoping reviews have been found to be suitable for synthesis of evidence in emerging fields where diversities of research methodologies have been used to define the landscape through a six-stage framework. The six-stage framework approach to scoping reviews includes identification of research questions, searching for relevant studies, selecting studies based on predetermined criteria, charting the studies, collating and summarizing findings, and consultations with relevant stakeholders to validate study findings. ${ }^{34}$ In this study, we did not include the sixth stage of consultation with stakeholders. However, our analyses and interpretations was informed by our own work in the field of T\&CM, during which we interacted with a diversity of stakeholders. ${ }^{27,35-38}$

\section{Eligibility Criteria and Search Strategies Inclusion Criteria}

Studies were included in this review if they fulfilled the following criteria: (i) published in a peer-reviewed journal; (ii) published in the English language; (iii) original research article on the use of T\&CM by adult cancer patients (aged 18 years and above) with any cancer types; (iv) the cancer patients in the study self-reported using at least one type of $\mathrm{T} \& \mathrm{CM}$ and were undergoing any of the known conventional cancer therapies, including chemotherapy, radiotherapy, surgery and palliative care; (v) the study design was stated and used either a quantitative or qualitative approach; (vi) data collection was carried out solely in a country in SSA; and (vii) the study outcome explicitly included the prevalence of use of T\&CM and other aspects of use related to the cancer diagnosis.

\section{Exclusion Criteria}

Studies were excluded from this review if they were: (i) published before 1st January 2000 or after 1st December 2019; and (ii) dissertations, theses or books.

\section{Search Strategies}

The study team discussed the proposed study, formulated the study questions and objectives, defined the issues of concern and identified key search terms. The search terms were developed based on each of the research questions guiding the review. An experienced medical librarian (AAK) conversant with systematic reviews, scoping reviews and literature search in the health and social sciences guided the formulation of the search terms. The librarian conducted the literature search for this scoping review. Three databases were searched: PubMed, Embase and Web of Science. The search terms, databases, dates of last search and number of articles retrieved from each database are detailed in Table 1.

In addition to the articles retrieved based on the search terms in Table 1, the authors reviewed the bibliographies of included articles for relevant references. Relevant articles identified from references of included articles were included for evaluation.

\section{Statistical Analyses}

We did not conduct statistical analyses on the data, mainly because of the heterogeneity of the study designs. Mean ages and standard deviations are reported as per each article without further synthesis. Medians were determined for prevalence of use and disclosure of use to healthcare professionals, and reported with their respective ranges (Table 2). Arithmetic means for prevalence and disclosure of use have not been calculated as these are often influenced by outliers or extreme values. 
Table I Search Terms and Databases Searched

\begin{tabular}{|c|c|c|}
\hline $\begin{array}{l}\text { Database, Search } \\
\text { Date }\end{array}$ & Search Terms & $\begin{array}{l}\text { Number of } \\
\text { Articles Retrieved }\end{array}$ \\
\hline $\begin{array}{l}\text { PubMed, Ist } \\
\text { December } 2019\end{array}$ & $\begin{array}{l}\text { "Traditional medicine" OR "Complementary medicine" OR "Alternative medicine" OR } \\
\text { Medicine, traditional[MeSH Terms] OR "Indigenous Medicine" OR Medicine, Indigenous[MeSH } \\
\text { Terms] OR "African traditional medicine" OR "Complementary therapies" OR } \\
\text { "Complimentary therapy" OR "Complementary Medicine" OR "Integrative Medicine" } \\
\text { AND } \\
\text { "Conventional treatment" OR "Conventional medicine" OR "Allopathic medicine" OR "Bio } \\
\text { medicine" OR Bio-medicine OR "Modern medicine" OR "Western medicine" } \\
\text { AND } \\
\text { Cancer* OR neoplas* OR carcinoma OR malignan* OR tumour OR tumor } \\
\text { AND } \\
\text { Adult* } \\
\text { AND } \\
\text { "Sub Saharan Africa" OR Sub-Saharan Africa OR "Africa South of the Sahara" OR SSA OR } \\
\text { Cameroon OR "Central African Republic" OR Chad OR Congo OR "Democratic Republic of } \\
\text { Congo" OR DRC OR "Equatorial Guinea" OR Gabon OR Sao "Tome and Principe" OR } \\
\text { Burundi OR Djibouti OR Eritrea OR Ethiopia OR Kenya OR Rwanda OR Somalia OR "South } \\
\text { Sudan" OR Sudan OR Tanzania OR Uganda OR Angola OR Botswana OR Lesotho OR Malawi } \\
\text { OR Mozambique OR Namibia OR "South Africa" OR Swaziland OR Zambia OR Zimbabwe OR } \\
\text { Benin OR Burkina Faso OR "Cabo Verde" OR "Cape Verde" OR "Ivory Coast" OR Gambia OR } \\
\text { Ghana OR Guinea OR Guinea-Bissau OR Liberia OR Mali OR Mauritania OR Niger OR Nigeria } \\
\text { OR Senegal OR "Sierra Leone" OR Togo } \\
\text { AND } \\
\text { PUBD: OI/OI/2000-0I/I2/20I9 }\end{array}$ & 8 \\
\hline $\begin{array}{l}\text { Web of Science, Ist } \\
\text { December } 2019\end{array}$ & $\begin{array}{l}\text { TS=((Traditional OR Complementary OR alternative OR indigenous OR “African traditional” } \\
\text { OR integrative) AND (medicine OR therap* OR treatment)) } \\
\text { AND } \\
\text { TS=((Conventional OR allopathic OR modern OR western) AND (medicine OR treatment) } \\
\text { OR Bio medicine OR Bio-medicine) } \\
\text { AND } \\
\text { TS=(Cancer* OR tumor OR tumour OR carcinoma OR malignan* OR neoplas*) } \\
\text { AND } \\
\text { TS=(Adult* OR "grown up" OR “I8 years and above" OR mature) } \\
\text { AND } \\
\text { TS=(Sub Saharan Africa OR Sub-Saharan Africa OR SSA OR Cameroon OR Central African } \\
\text { Republic OR Chad OR Congo OR Democratic Republic of Congo OR DRC OR Equatorial } \\
\text { Guinea OR Gabon OR Sao Tome and Principe OR Burundi OR Djibouti OR Eritrea OR } \\
\text { Ethiopia OR Kenya OR Rwanda OR Somalia OR South Sudan OR Sudan OR Tanzania OR } \\
\text { Uganda OR Angola OR Botswana OR Lesotho OR Malawi OR Mozambique OR Namibia OR } \\
\text { South Africa OR Swaziland OR Zambia OR Zimbabwe OR Benin OR Burkina Faso OR Cabo } \\
\text { Verde OR Cape Verde OR Ivory Coast OR Gambia OR Ghana OR Guinea OR Guinea-Bissau } \\
\text { OR Liberia OR Mali OR Mauritania OR Niger OR Nigeria OR Senegal OR Sierra Leone OR } \\
\text { Togo) } \\
\text { AND } \\
\text { PUBD: OI/OI/2000-0I/I2/20I9 }\end{array}$ & 10 \\
\hline $\begin{array}{l}\text { EMBASE, Ist } \\
\text { December } 2019\end{array}$ & $\begin{array}{l}\text { ((Traditional OR Complementary OR alternative OR indigenous OR African traditional OR } \\
\text { integrative) AND (medicine OR therap* OR treatment)) } \\
\text { AND }\end{array}$ & 78 \\
\hline
\end{tabular}

(Continued) 
Table I (Continued).

\begin{tabular}{|c|c|c|}
\hline $\begin{array}{l}\text { Database, Search } \\
\text { Date }\end{array}$ & Search Terms & $\begin{array}{l}\text { Number of } \\
\text { Articles Retrieved }\end{array}$ \\
\hline & $\begin{array}{l}\text { ((Conventional OR allopathic OR modern OR western AND (medicine OR treatment) OR Bio } \\
\text { medicine OR Bio-medicine) } \\
\text { AND } \\
\text { Cancer* OR tumor OR tumour OR carcinoma OR malignan* OR neoplas* } \\
\text { AND } \\
\text { Adult* OR "grown up" OR "I8 years and above" OR mature } \\
\text { AND } \\
\text { "Sub Saharan Africa" OR "Sub-Saharan Africa" OR Cameroon OR "Central African Republic" } \\
\text { OR Chad OR Congo OR "Democratic Republic of Congo" OR DRC OR "Equatorial Guinea" } \\
\text { OR Gabon OR "Sao Tome and Principe" OR Burundi OR Djibouti OR Eritrea OR Ethiopia OR } \\
\text { Kenya OR Rwanda OR Somalia OR "South Sudan" OR Sudan OR Tanzania OR Uganda OR } \\
\text { Angola OR Botswana OR Lesotho OR Malawi OR Mozambique OR Namibia OR "South Africa" } \\
\text { OR Swaziland OR Zambia OR Zimbabwe OR Benin OR "Burkina Faso" OR "Cabo Verde" OR } \\
\text { "Cape Verde" OR "Ivory Coast" OR Gambia OR Ghana OR Guinea OR Guinea-Bissau OR } \\
\text { Liberia OR Mali OR Mauritania OR Niger OR Nigeria OR Senegal OR "Sierra Leone" OR Togo } \\
\text { AND } \\
\text { PUBD: 0I/OI/2000-0I/I2/20I9 }\end{array}$ & \\
\hline
\end{tabular}

\section{Results}

In total, 96 articles were retrieved from three databases into separate reference managers. The reference manager files (Endnotes) were merged and duplicate articles were identified; nine duplicate articles were removed. The titles and abstracts of the remaining articles were reviewed independently by ADM and CA for suitability based on the predefined inclusion criteria. Whenever there was lack of clarity regarding eligibility, all the authors read the particular articles $(\mathrm{n}=3)$, met and agreed by consensus based on the eligibility criteria. References of the selected articles were screened for suitable articles; three articles were added from a review of references of selected articles. ADM critically read 23 full-text articles to extract data to answer the preset research questions. Altogether, 12 full-text articles were included in this review (Figure 1). These articles contributed a sample size of 2225 cancer patients with different cancer types. The majority of the articles were from Nigeria $(33.3 \%, n=4)$ and Uganda (25\%, $\mathrm{n}=3$ ) (Table 2).

\section{Prevalence of T\&CM Use}

The prevalence of use of T\&CM varied within and across countries. The median prevalence of use of T\&CM was $60.0 \%$ (range: $14.1-79.0 \%$ ).

\section{Disclosure of Use of T\&CM to Healthcare Professionals}

In seven of the 12 articles, there was information on disclosure of use of T\&CM to the attending healthcare professionals. The median percent disclosure was $32.7 \%$, (range: 15.3-85.7\%). Again, percent disclosure varied within and between countries. Only a few articles, including those by Mwaka et al, Ong'udi et al, and Ezeome and Anarado, reported on patients' reasons for non-disclosure. ${ }^{3,27,39}$ The most common reasons for non-disclosure were that the doctor did not ask, the doctor would rebuke them for use of $\mathrm{T} \& \mathrm{CM}$, and the doctors do not know much about T\&CM and so there is no need to share the issue of use with them.

\section{Types of T\&CM Used by Cancer Patients}

Two of the studies included did not explicitly state the types of T\&CM used by their study participants. In the other ten studies, the T\&CMs used by cancer patients included herbs of various types, healing prayers or spiritual approaches, divination, massage, meditation and animal products e.g. python fat. The reported prevalence of use of each product varied among the studies. In general, herbal products comprising various plant parts prepared in different forms constituted the most common herbal medicinal products in use. 


\begin{tabular}{|c|c|c|}
\hline 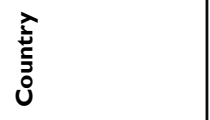 & 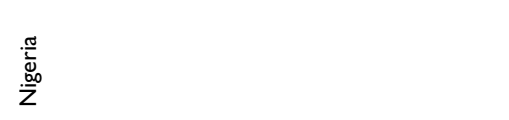 & 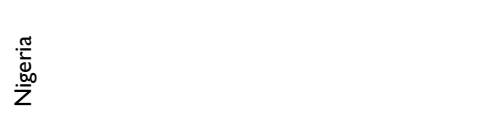 \\
\hline 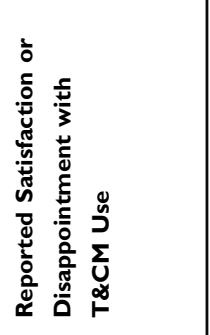 & 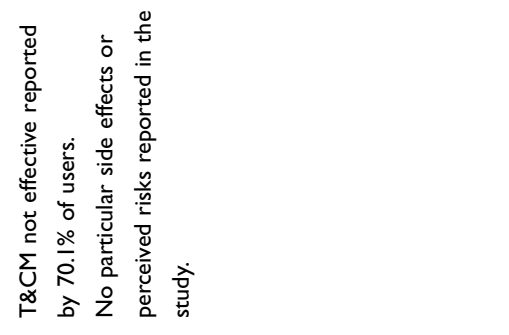 & 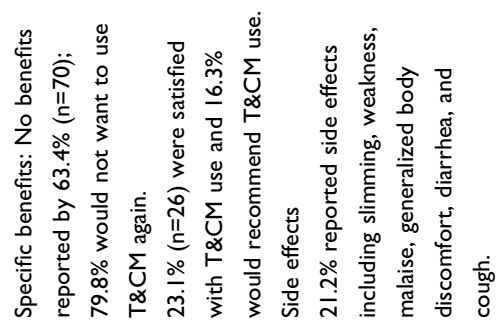 \\
\hline 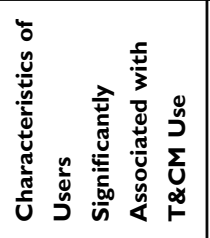 & 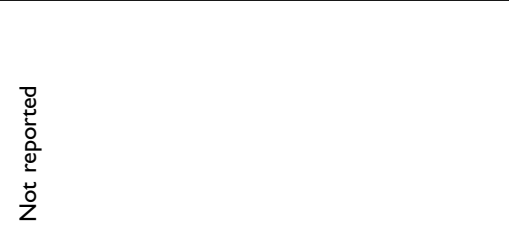 & 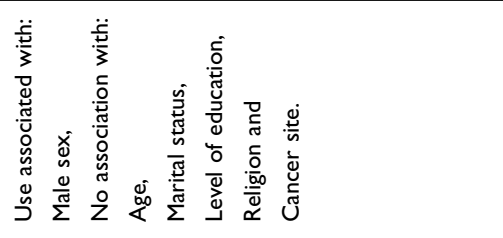 \\
\hline 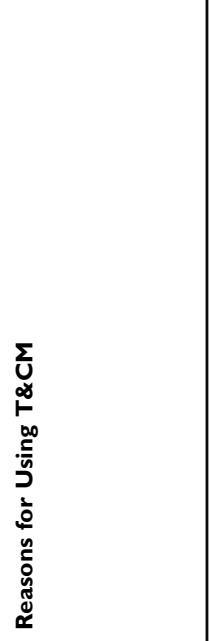 & 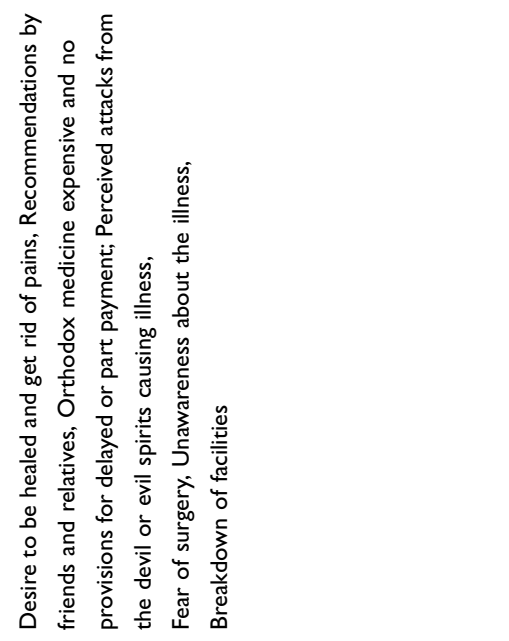 & 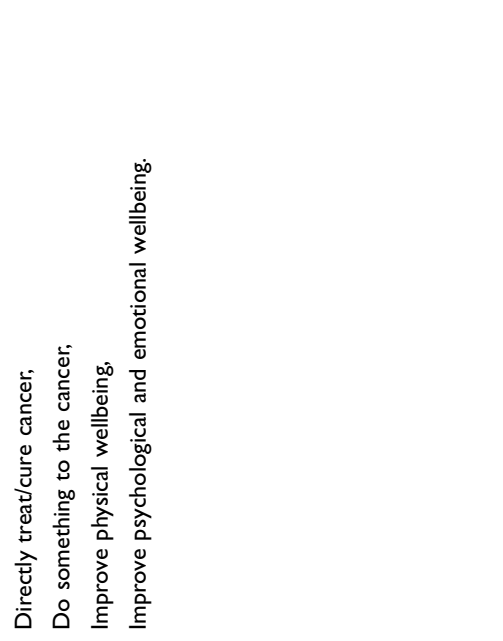 \\
\hline 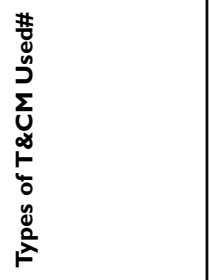 & 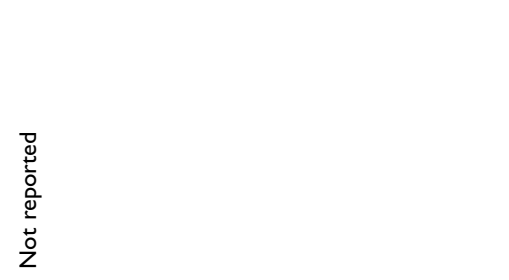 & 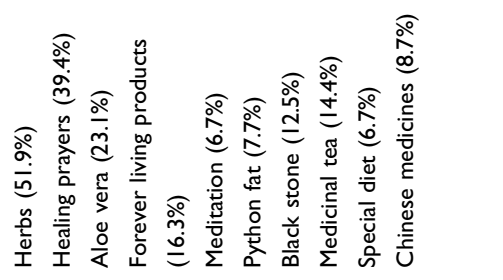 \\
\hline 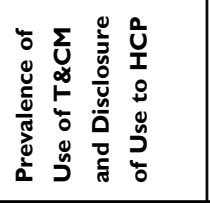 & 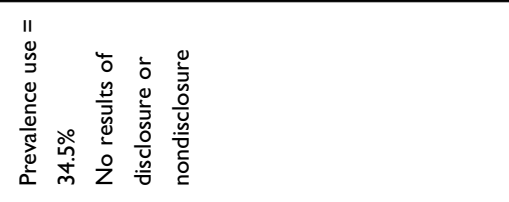 & 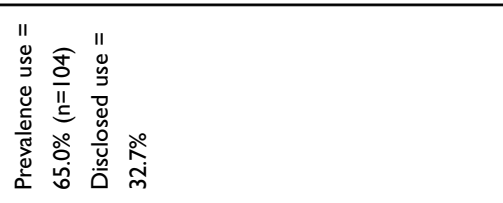 \\
\hline 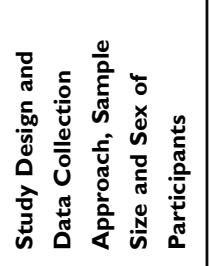 & 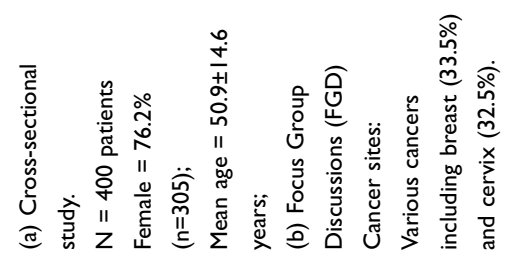 & 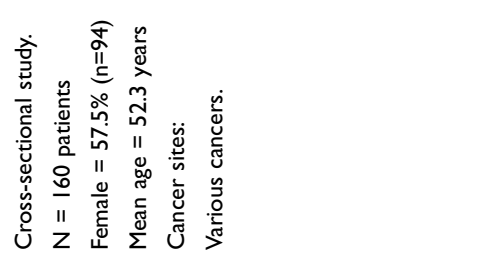 \\
\hline 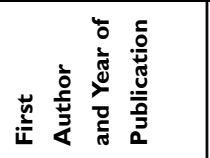 & 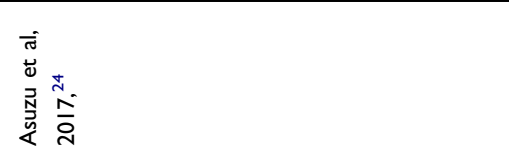 & 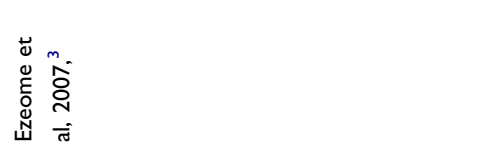 \\
\hline
\end{tabular}




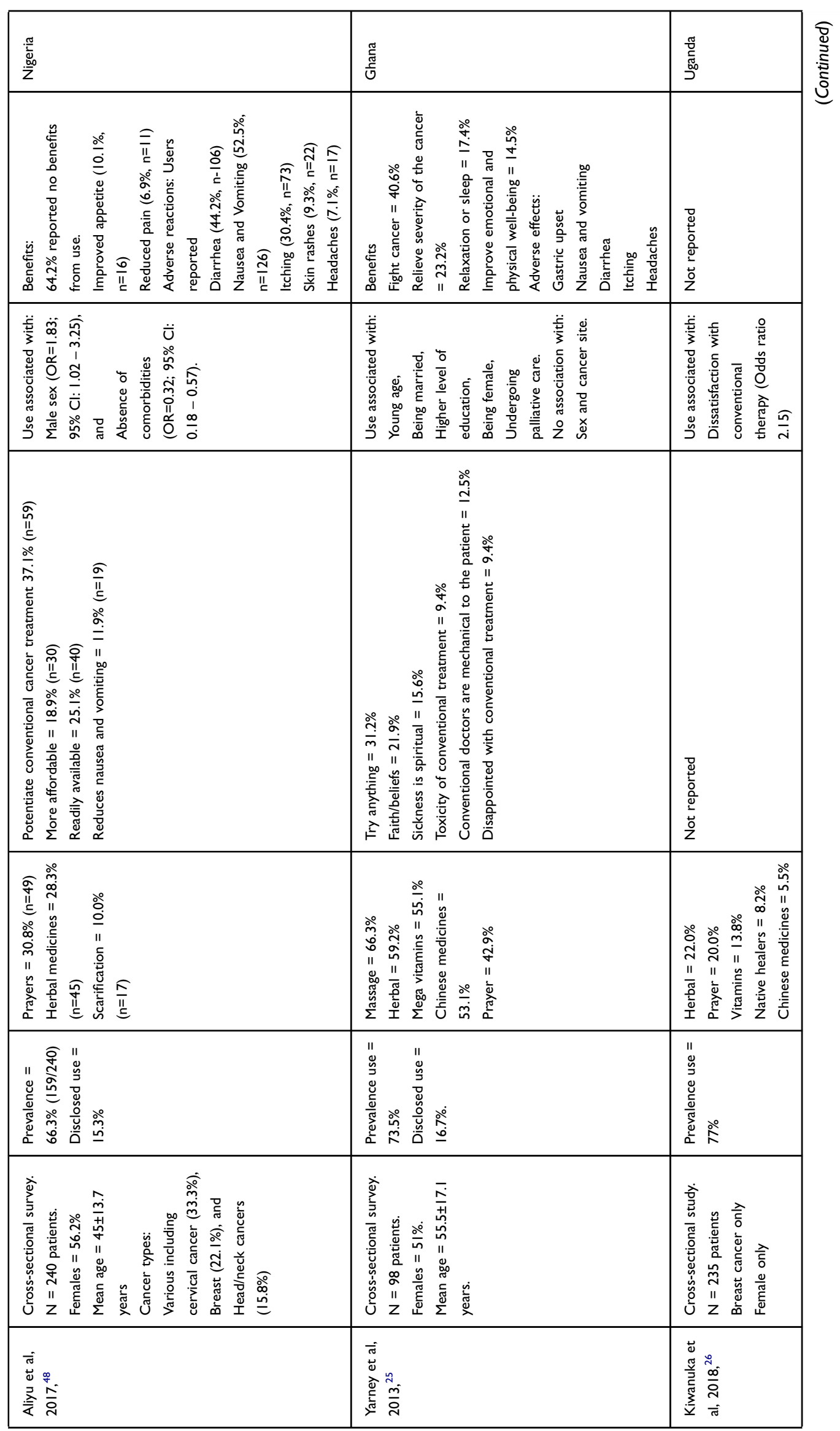




\begin{tabular}{|c|c|c|c|c|}
\hline نे & 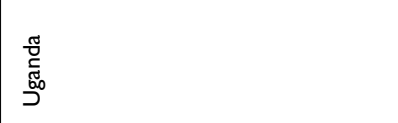 & 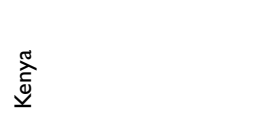 & 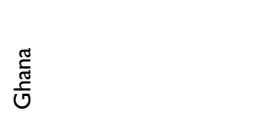 & 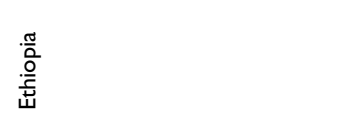 \\
\hline 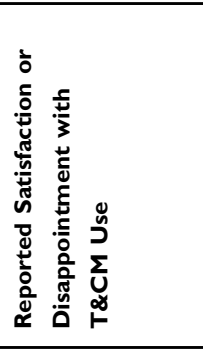 & 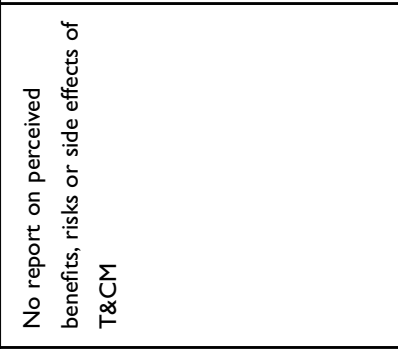 & 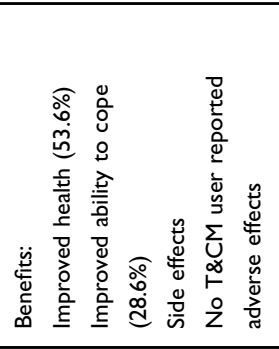 & 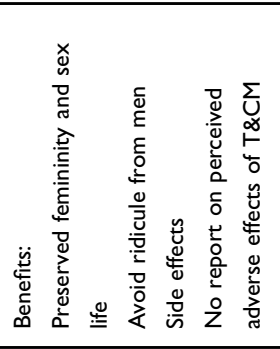 & 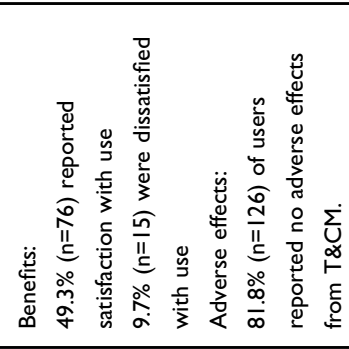 \\
\hline 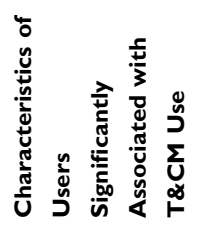 & 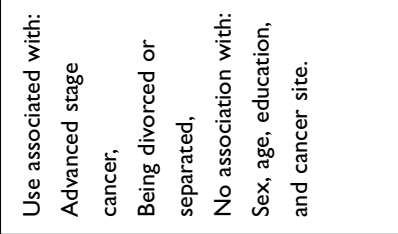 & 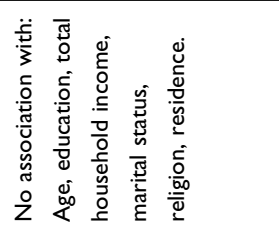 & 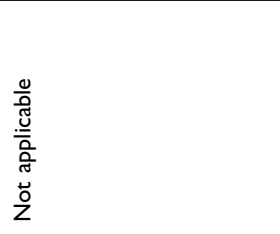 & 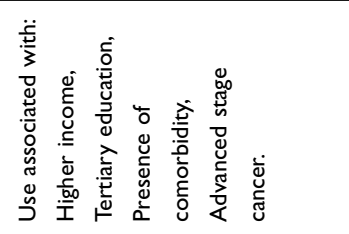 \\
\hline 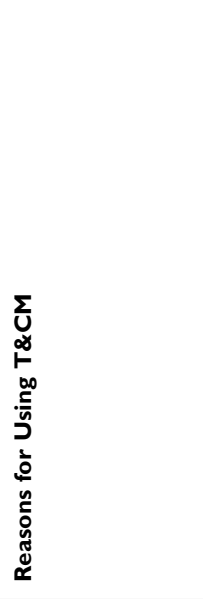 & 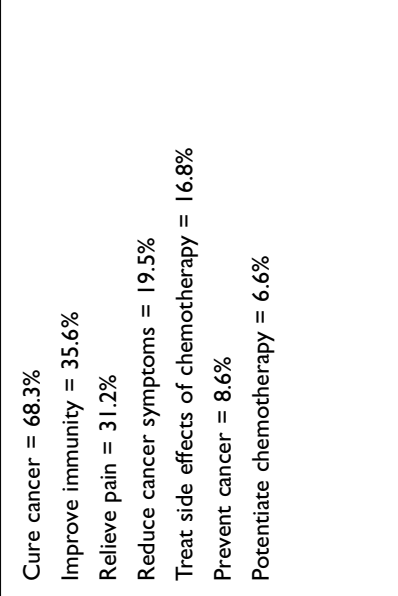 & 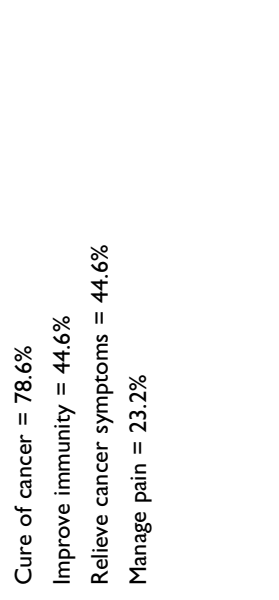 & 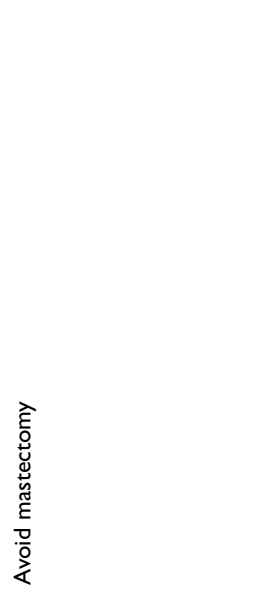 & 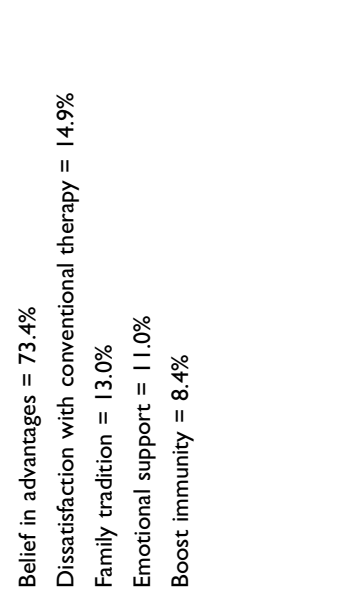 \\
\hline 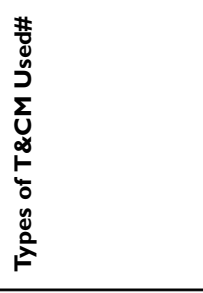 & 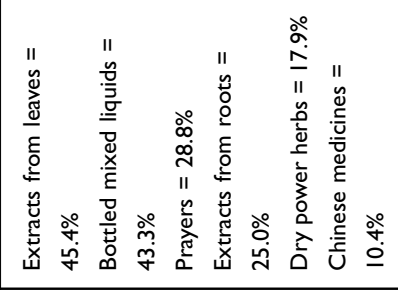 & 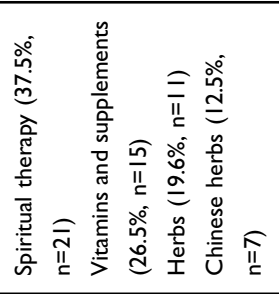 & 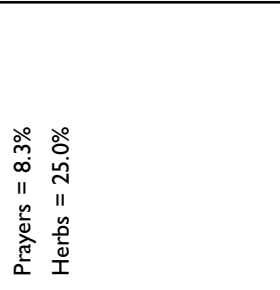 & 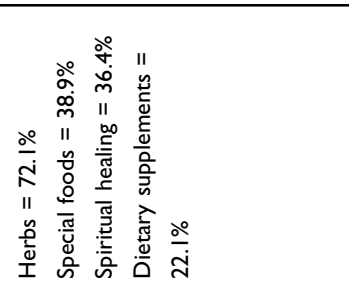 \\
\hline 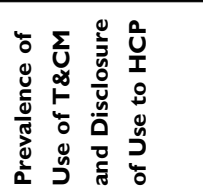 & 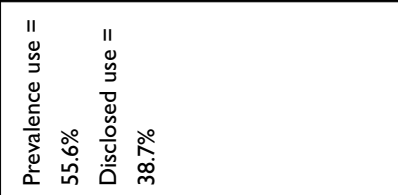 & 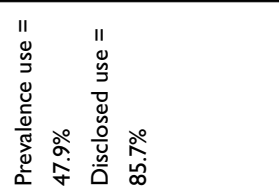 & 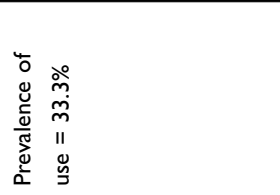 & 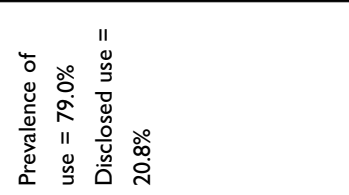 \\
\hline 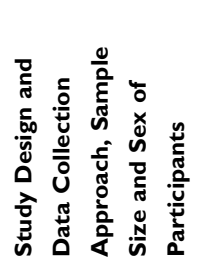 & 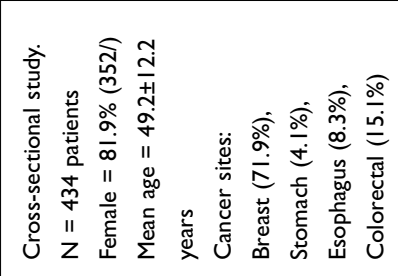 & 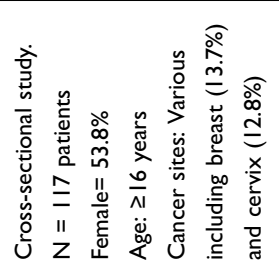 & 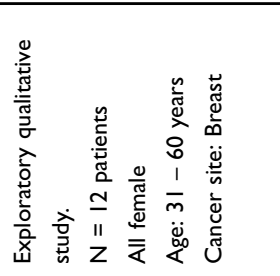 & 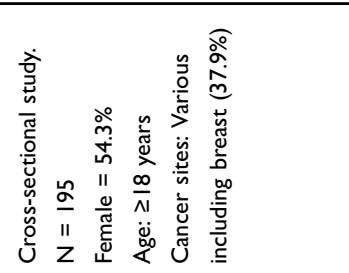 \\
\hline 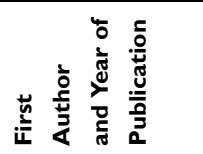 & 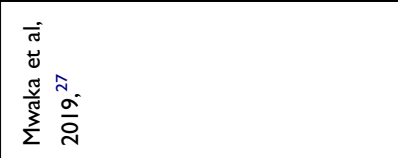 & 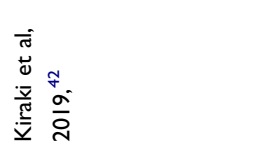 & 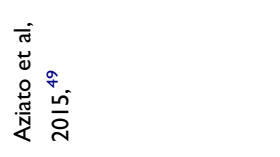 & 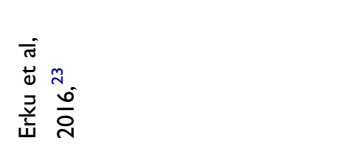 \\
\hline
\end{tabular}




\begin{tabular}{|c|c|c|}
\hline 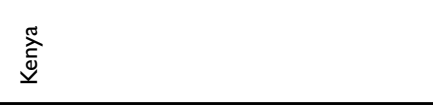 & 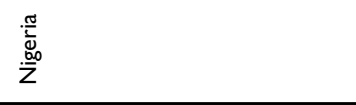 & $\begin{array}{l}\text { 芯 } \\
\text { 离 }\end{array}$ \\
\hline 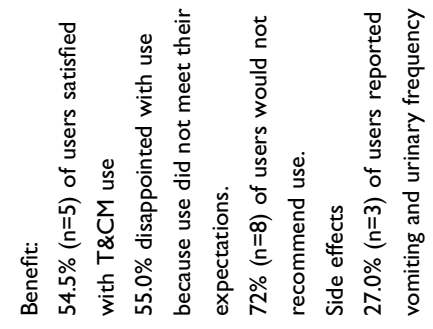 & 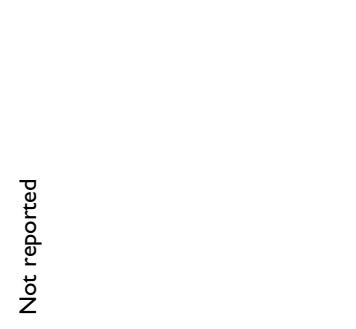 & 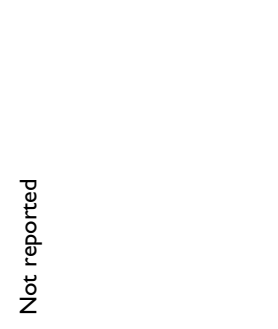 \\
\hline 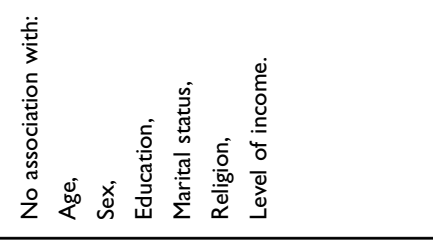 & 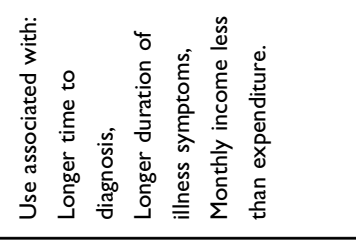 & 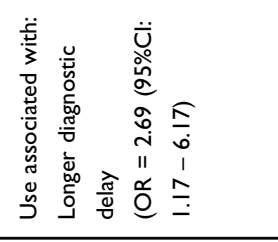 \\
\hline 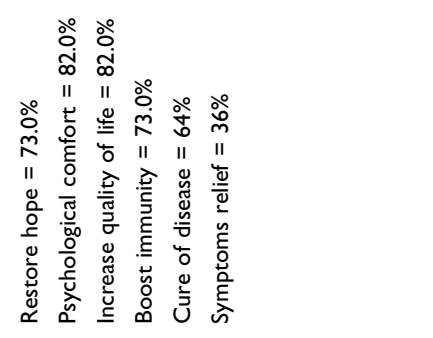 & 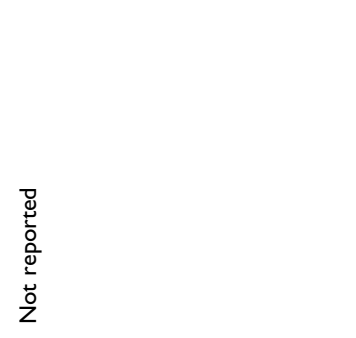 & 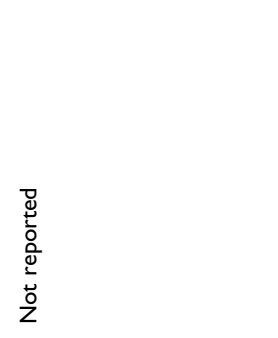 \\
\hline 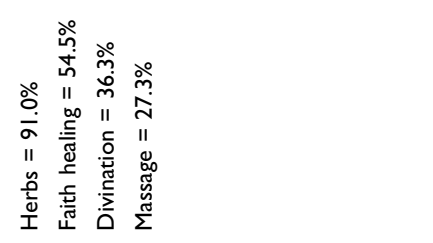 & 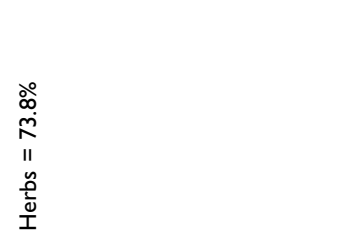 & 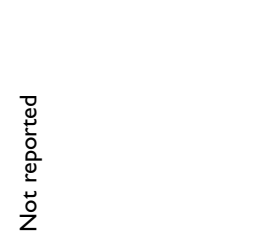 \\
\hline 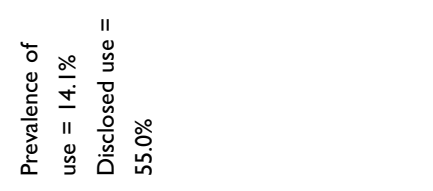 & 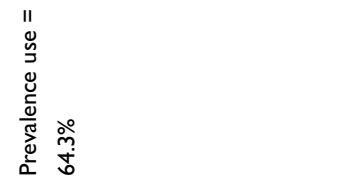 & 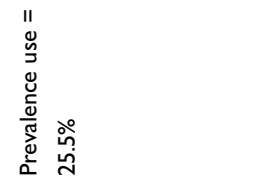 \\
\hline 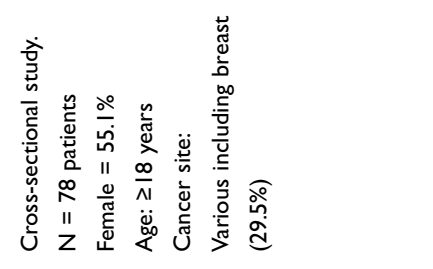 & 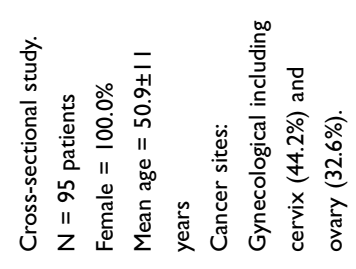 & 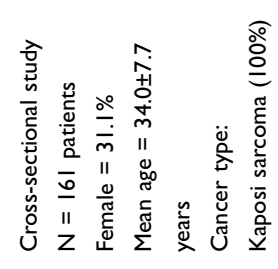 \\
\hline 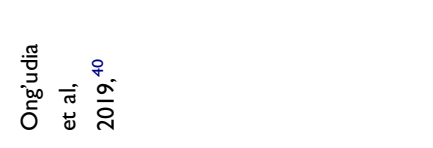 & 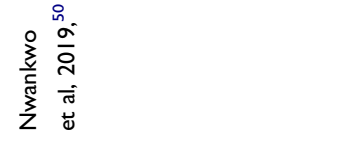 & 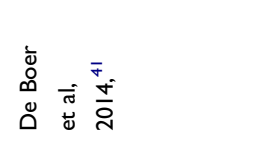 \\
\hline
\end{tabular}




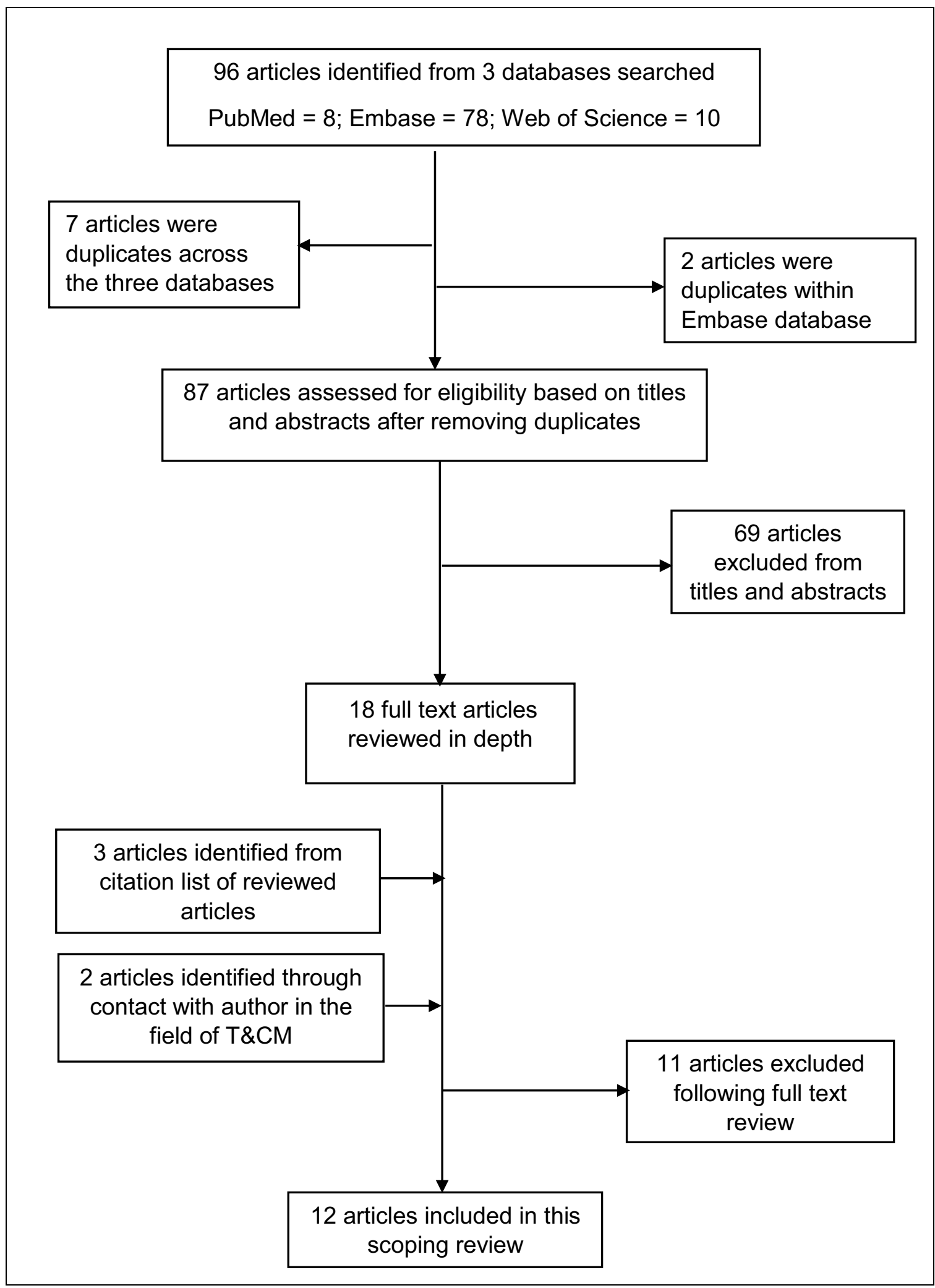

Figure I Study evaluation flowchart. 


\section{Reasons for Use of T\&CM}

In eight of the 12 articles, the authors reported on the reasons for cancer patients using $\mathrm{T} \& \mathrm{CM}$. The reasons were many and varied, including the wish to get rid of cancer symptoms, especially pain, treat/cure cancer, improve physical and psychological well-being, treat toxicity of conventional cancer therapies and improve immunity. Fear of surgery, concern with the devil's influence in the disease process and the high cost of conventional cancer therapies were also reported reasons for the use of T\&CM (Table 2).

\section{Safety and Risks of T\&CM Among Cancer Patients}

We did not find any studies that objectively evaluated safety and risk profiles of T\&CM use among cancer patients in SSA. However, there were reports on perceived benefits and side effects of T\&CM use. Perceived benefits from T\&CM use included improved appetite, reductions in pain and other cancer symptoms, relaxation and improved sleep, improved emotional and physical well-being, improved ability to cope with illness, and preserved femininity and sex life. Perceived side effects of concern included loss of weight, general weakness and malaise, nausea and vomiting, diarrhea, itching, skin rashes, headaches and increased urinary frequency. Cancer patients who self-reported not using T\&CM said they would not use them because of side effects and perceived risks to internal organs, including the kidneys and liver. In addition, patients who used T\&CM but did not experience any benefits commensurate to their expectations would neither wish to use nor recommend the use of T\&CM to other patients in the future.

\section{Characteristics of Cancer Patients Who Use T\&CM}

There was no consistent pattern of association between particular patients' characteristics, disease features and health system factors with the use of T\&CM. Eleven of the studies included information on factors that were statistically significantly associated with the use of T\&CM. While some studies found an association between sex and T\&CM use, other studies did not find such associations. Other patient factors evaluated included age, marital status, educational attainment, income level and religion. Again, the associations were not consistent across studies. Disease factors evaluated included cancer site and stage at diagnosis. Cancer site was not associated with the use of $\mathrm{T} \& \mathrm{CM}$ in any of the studies.; however, cancer stage at diagnosis and presence of comorbidities were consistently positively associated with the use of T\&CM. Similarly, studies that evaluated time to presentation and diagnosis found statistically significant associations with the use of T\&CM. For example, in the study by De Boer et al, ${ }^{40}$ there was a significant association between the use of $\mathrm{T} \& \mathrm{CM}$ and diagnostic delay after adjusting for gender, age, income, ability to pay out of pocket and previous HIV clinic attendance (OR 2.69, $p=0.020,95 \%$ CI: 1.17-6.17).

\section{Discussion}

We found that $14.1-79.0 \%$ of cancer patients in the included studies reported the use of T\&CM for the treatment of their cancers. However, the majority of patients never disclosed the use of T\&CM to their healthcare professionals. The disclosure proportion exceeded $50 \%$ in only two studies $(85.7 \%$ in Monicah et al and $55.0 \%$ in Ong'udi et al). ${ }^{39,41}$ The reasons for non-disclosure included fear of rebuke and stigmatization by healthcare professionals, not being asked about T\&CM use, and perceived low knowledge of healthcare professionals on $\mathrm{T} \& \mathrm{CM}$ and therefore there being no need to discuss it with them. A high prevalence of use of $\mathrm{T} \& \mathrm{CM}$ has been reported in recent review of studies on T\&CM use among patients with various illnesses in low- and middle-income countries, including in SSA. ${ }^{1,42}$ Doctors and nurses in SSA need to take note of the high prevalence and low disclosure of use of T\&CM among cancer patients in their care. They perhaps need to proactively engage with patients regarding the use of T\&CM in order to help the patients make appropriate decisions and minimize possible side effects from concurrent use of T\&CM with conventional cancer medicines. This may require that the healthcare professionals learn more about T\&CM so that they can engage meaningfully with patients. Studies in medical schools in SSA reveal that the majority of medical students and their teachers are willing to accept integration of T\&CM into the medical school curricula. ${ }^{12,35,36,43}$ However, only a few medical schools in SSA have incorporated aspects of T\&CM into their curricula. ${ }^{44}$ Therefore, there is an urgent need to further explore the integration process in a bid to promote patient safety and harmonious relationships between the biomedical health systems and traditional health practices in SSA. 
Cancer patients used T\&CM for various reasons and expectations, including cure of cancers, management of cancer symptoms, boosting the immune system, improving physical and psychological well-being, fear of surgery, recommendation by friends and family, and trust in T\&CM providers. The majority of the patients who derived some subjective benefits from T\&CM would use them again and recommend them to other patients. The use of T\&CM is therefore likely to continue alongside conventional cancer treatments, mainly because it has long been part of the culture of the people and the patients trust T\&CM providers, and because of convenient methods of payment for T\&CM. ${ }^{2,45}$ The studies reviewed herein showed that patients also use T\&CM because of dissatisfaction with conventional medical care, fear of surgery and multiple side effects of conventional cancer medicines, and because T\&CMs are readily available and cheaper than conventional medicines. ${ }^{23-25,46,47} \mathrm{~T} \& \mathrm{CM}$ practitioners often provide payment schedules that are favorable to the patients. These include part payments, payments after a favorable outcome, e.g. cure has been achieved, and payments in kind. ${ }^{48,49}$ These attractive payment schedules are not available in biomedical facilities. Improved care for cancers could involve mainstreaming the use of T\&CM and increasing access to conventional cancer therapies by countries in SSA.

We found that cancer patients used several types of $\mathrm{T} \& \mathrm{CM}$, including herbs, prayers, dietary supplements and vitamins. ${ }^{3,27,46,50}$ However, none of the studies evaluated patients' perceptions of how the T\&CMs they used work on cancer. A few of the studies evaluated patients' perceptions of whether the T\&CMs used benefited them or caused them unwanted effects. ${ }^{3,25,39,46}$ Insights into how these T\&CMs are perceived to work, or indeed do work, could guide investigations into their mechanisms of action, active ingredients and efficacy, as well as adverse effects of the T\&CMs. Efforts to counsel and/or to reverse the perceptions and beliefs of patients regarding T\&CM that could be dangerous to their health, either directly or through interactions with conventional medicines, require adequate understanding of the patients' perceived mechanism of action and benefits of particular T\&CMs. Therefore, more qualitative studies are needed to explore the different types of T\&CM used for treatment of cancer, and to gain insights into patients' perceptions and beliefs regarding mechanisms of action, how benefits arise and side effect profiles of particular T\&CMs.

In this review, we did not find any data on the objective assessment of safety and risk of the T\&CMs used. Four of the
12 studies reviewed reported on perceived side effects of T\&CM. Reported side effects included increased urinary frequency, nausea and vomiting, diarrhea, loss of weight, generalized weakness and skin rashes. ${ }^{3,25,39,46}$ Most of these symptoms could be a direct result of the cancer and/ or side effects of the conventional cancer therapies, rather than side effects of T\&CM. There is an urgent need for welldesigned efficacy, effectiveness and safety studies on T\&CM use in SSA, especially among patients with chronic diseases who are likely to use T\&CM for a long enough period to develop cumulative unwanted effects.

This study has some limitations. First, we included only studies published in the English language and yet a number of countries in SSA could have used other languages, e.g. French, in their publications. Second, a systematic review with pooled analyses of data from the individual critically appraised studies could have provided deeper understanding of the subject matter. However, we chose a scoping review because there are few studies in the field of T\&CM and cancer. The scoping review approach is more suitable to synthesize available knowledge and guide future studies.

\section{Conclusion}

The majority of cancer patients undergoing conventional cancer therapies in SSA use T\&CMs of various types concurrently with conventional cancer therapies. The majority of cancer patients who use T\&CM do not disclose the use of T\&CM to the healthcare professionals providing them with conventional cancer therapies. Nondisclosure of the use of T\&CM concurrently with conventional cancer treatments to the healthcare professionals potentially exposes patients to danger, including side effects of T\&CM and interactions between the drugs and herbs. Cancer patients need to be encouraged to disclose the use of T\&CM to their healthcare professionals, who need to be more courteous when they deal with matters of T\&CM.

\section{Acknowledgment}

The authors wish to thank Professor Sunita Vohra for valuable guidance during development of research questions for this review study. We also thank the library staff who supported the literature search.

\section{Author Contributions}

All authors made substantial contributions to conception and design, acquisition of data, or analysis and interpretation of 
data; took part in drafting the article or revising it critically for important intellectual content; gave final approval of the version to be published; and agree to be accountable for all aspects of the work.

\section{Disclosure}

The authors report no conflicts of interest in this work.

\section{References}

1. James PB, Wardle J, Steel A, Adams J. Traditional, complementary and alternative medicine use in Sub-Saharan Africa: a systematic review. BMJ Global Health. 2018;3(5):e000895. doi:10.1136/ bmjgh-2018-000895

2. Rutebemberwa E, Lubega M, Katureebe SK, Oundo A, Kiweewa F, Mukanga D. Use of traditional medicine for the treatment of diabetes in Eastern Uganda: a qualitative exploration of reasons for choice. BMC Int Health Hum Rights. 2013;13:1. doi:10.1186/1472-698X-131

3. Ezeome ER, Anarado AN. Use of complementary and alternative medicine by cancer patients at the University of Nigeria Teaching Hospital, Enugu, Nigeria. BMC Complement Altern Med. 2007;7:28. doi:10.1186/1472-6882-7-28

4. Lunyera J, Wang D, Maro V, et al. Traditional medicine practices among community members with diabetes mellitus in Northern Tanzania: an ethnomedical survey. BMC Complement Altern Med. 2016;16(1):282. doi:10.1186/s12906-016-1262-2

5. Liwa AC, Smart LR, Frumkin A, Epstein H-AB, Fitzgerald DW, Peck RN. Traditional herbal medicine use among hypertensive patients in Sub-Saharan Africa: a systematic review. Curr Hypertens Rep. 2014;16(6):437. doi:10.1007/s11906-014-0437-9

6. WHO. Traditional Medicine Strategy 2002-2005. Geneva: World Health Organization; 2002.

7. WHO. WHO traditional medicine strategy: 2014-2023. Available from: http://appswhoint/iris/bitstream/10665/92455/1/ 9789241506090_engpdf?ua=1. Accessed March 23, 2015.

8. WHO. Beijing declaration. 2008. Available from: http://wwwwhoint/ medicines/areas/traditional/TRM_BeijingDeclarationENpdf?ua=1. Accessed March 23, 2015.

9. Kasilo OM, Trapsida J-M, Mwikisa Ngenda C, Lusamba-Dikassa PS; régional pour l'Afrique $\mathrm{B}$, Organization $\mathrm{WH}$. An overview of the traditional medicine situation in the African region. Afr Health Monit. 2010:7-15.

10. WHO. Legal status of traditional medicine and complementary/alternative medicine: a worldwide review. 2001. Available from: https:// appswhoint/medicinedocs/pdf/h2943e/h2943epdf. Accessed April 10, 2020.

11. Republic of South Africa. Traditional health practitioners act, 2007. No. 22 of 2007. Republic of South Africa. Available from: https:// wwwgovza/sites/default/files/gcis_document/201409/a22-07pdf. Accessed April 10, 2020. 2008.

12. Ameade EP, Amalba A, Helegbe G, Mohammed B. Medical students' knowledge and attitude towards complementary and alternative medicine - a survey in Ghana. J Tradit Complement Med. 2016;6(3):230 236. doi:10.1016/j.jtcme.2015.03.004

13. Bray F, Ferlay J, Soerjomataram I, Siegel RL, Torre LA, Jemal A. Global cancer statistics 2018: GLOBOCAN estimates of incidence and mortality worldwide for 36 cancers in 185 countries. CA Cancer J Clin. 2018;68(6):394-424.

14. Bray F, Jemal A, Grey N, Ferlay J, Forman D. Global cancer transitions according to the Human Development Index (2008-2030): a population-based study. Lancet Oncol. 2012;13(8):790-801. doi:10.1016/S1470-2045(12)70211-5
15. Parkin DM, Bray F, Ferlay J, Jemal A. Cancer in Africa 2012. Cancer Epidemiol Biomarker Prev. 2014;23(6):953-966. doi:10.1158/10559965.EPI-14-0281

16. Wabinga HR, Nambooze S, Amulen PM, Okello C, Mbus L, Parkin DM. Trends in the incidence of cancer in Kampala, Uganda 1991-2010. Int $J$ Cancer. 2014;135(2):432-439. doi:10.1002/ ijc. 28661

17. Mwaka AD, Garimoi CO, Were EM, Roland M, Wabinga H, Lyratzopoulos G. Social, demographic and healthcare factors associated with stage at diagnosis of cervical cancer: cross-sectional study in a tertiary hospital in Northern Uganda. BMJ Open. 2016;6(1): e007690. doi:10.1136/bmjopen-2015-007690

18. Pace LE, Mpunga T, Hategekimana V, et al. Delays in breast cancer presentation and diagnosis at two rural cancer referral centers in Rwanda. Oncologist. 2015;20(7):780-788. doi:10.1634/theoncologist.2014-0493

19. Ibrahim A, Rasch V, Pukkala E, Aro AR. Predictors of cervical cancer being at an advanced stage at diagnosis in Sudan. Int $J$ Women Health. 2011;3:385-389. doi:10.2147/IJWH.S21063

20. Wabinga H, Ramanakumar AV, Banura C, Luwaga A, Nambooze S, Parkin DM. Survival of cervix cancer patients in Kampala, Uganda: 1995-1997. Br $J$ Cancer. 2003;89(1):65-69. doi:10.1038/sj. bjc. 6601034

21. Chokunonga E, Ramanakumar AV, Nyakabau AM, et al. Survival of cervix cancer patients in Harare, Zimbabwe, 1995-1997. Int $J$ Cancer. 2004;109(2):274-277. doi:10.1002/ijc.11670

22. Gondos A, Brenner H, Wabinga H, Parkin DM. Cancer survival in Kampala, Uganda. Br J Cancer. 2005;92(9):1808-1812. doi:10.1038/ sj.bjc. 6602540

23. Erku DA. Complementary and alternative medicine use and its association with quality of life among cancer patients receiving chemotherapy in Ethiopia: a cross-sectional study. Evid Based Complement Alternat Med. 2016;2016:8. doi:10.1155/2016/2809875

24. Asuzu CC, Elumelu-Kupoluyi T, Asuzu MC, Campbell OB, AkinOdanye EO, Lounsbury D. A pilot study of cancer patients' use of traditional healers in the Radiotherapy Department, University College Hospital, Ibadan, Nigeria. Psycho Oncol. 2017;26(3):369376. doi:10.1002/pon.4033

25. Yarney J, Donkor A, Opoku SY, et al. Characteristics of users and implications for the use of complementary and alternative medicine in Ghanaian cancer patients undergoing radiotherapy and chemotherapy: a cross- sectional study. BMC Complement Altern Med. 2013;13 (1):16. doi:10.1186/1472-6882-13-16

26. Kiwanuka F. Complementary and alternative medicine use: influence of patients' satisfaction with medical treatment among breast cancer patients at Uganda Cancer Institute. Adv Biosci Clin Med. 2018;6 (1):24-29. doi:10.7575/aiac.abcmed.v.6n.1p.24

27. Mwaka AD, Mangi SP, Okuku FM. Use of traditional and complementary medicines by cancer patients at a national cancer referral facility in a low-income country. Eur J Cancer Care (Engl). 2019;28 (6):e13158. doi:10.1111/ecc.13158

28. Nabukenya I. Acute and sun-acute toxicity of ethanolic and aqueous extracts of tephrosia vogelii, Vernonia amygdalina and Senna occidentalis in Rodents. Altern Integr Med. 2014;3(3).

29. Kiguba R, Ononge S, Karamagi C, Bird S. Herbal medicine use and linked suspected adverse drug reactions in a prospective cohort of Ugandan inpatients. BMC Complement Altern Med. 2016;16(145). doi:10.1186/s12906-016-1125-x

30. Auerbach B, Reynolds S, Lamorde M, et al. Traditional herbal medicine use associated with liver fibrosis in Rural Rakai, Uganda. PLoS One. 2012;7(11):e41737. doi:10.1371/journal.pone.0041737

31. Anywar G, Van't Klooster C, Byamukama R, et al. Medicinal plants used in the treatment and prevention of malaria in Cegere SubCounty, Northern Uganda. Ethnobot Res Appl. 2016;14:505-516. doi:10.17348/era.14.0.505-516 
32. Lubinga SJ, Kintu A, Atuhaire J, Asiimwe S. Concomitant herbal medicine and Antiretroviral Therapy (ART) use among HIV patients in Western Uganda: a cross-sectional analysis of magnitude and patterns of use, associated factors and impact on ART adherence. AIDS Care. 2012;24(11):1375-1383. doi:10.1080/09540121.2011. 648600

33. Block KI. Significance of natural product interactions in oncology. Integr Cancer Ther. 2012;12(1):4-6. doi:10.1177/1534735412467368

34. Arksey H, O'Malley L. Scoping studies: towards a methodological framework. Int J Soc Res Methodol. 2005;8(1):19-32. doi:10.1080/ 1364557032000119616

35. Mwaka AD, Tusabe G, Garimoi CO, Vohra S, Ibingira C. Integration of traditional and complementary medicine into medical school curricula: a survey among medical students in Makerere University, Uganda. BMJ Open. 2019;9(9):e030316. doi:10.1136/bmjopen2019-030316

36. Mwaka AD, Tusabe G, Orach Garimoi C, Vohra S. Turning a blind eye and a deaf ear to traditional and complementary medicine practice does not make it go away: a qualitative study exploring perceptions and attitudes of stakeholders towards the integration of traditional and complementary medicine into medical school curriculum in Uganda. BMC Med Educ. 2018;18(1):310. doi:10.1186/ s12909-018-1419-4

37. Mwaka AD, Okello ES, Orach CG. Barriers to biomedical care and use of traditional medicines for treatment of cervical cancer: an exploratory qualitative study in northern Uganda. Eur $J$ Cancer Care (Engl). 2015;24(4):503-513. doi:10.1111/ecc.12211

38. Abbo C, Ekblad S, Waako P, Okello E, Musisi S. The prevalence and severity of mental illnesses handled by traditional healers in two districts in Uganda. Afr Health Sci. 2009;9(2).

39. Ong'udi M, Mutai P, Weru I. Study of the use of complementary and alternative medicine by cancer patients at Kenyatta National Hospital, Nairobi, Kenya. J Oncol Pharm Pract. 2019;25(4):918928. doi:10.1177/1078155218805543

40. De Boer C, Niyonzima N, Orem J, Bartlett J, Zafar SY. Prognosis and delay of diagnosis among Kaposi's sarcoma patients in Uganda: a cross-sectional study. Infect Agent Cancer. 2014;9(17):1750-9378. doi:10.1186/1750-9378-9-17
41. Monicah K, Mbugua G, Mburugu R. Use of complementary and alternative medicine among cancer patients in Meru County, Kenya. Int J Prof Pract. 2019;7(1):24-33.

42. Hill J, Mills C, Li Q, Smith JS. Prevalence of traditional, complementary, and alternative medicine use by cancer patients in low income and lower-middle income countries. Glob Public Health. 2019;14(3):418-430. doi:10.1080/17441692.2018.1534254

43. Kazembe TC, Musekiwa M. Inclusion of traditional medicine in the school curriculum in Zimbabwe: a case study. Eurasian J Anthropol. 2011;2(2):54-69.

44. Chitindingu E, George G, Gow J. A review of the integration of traditional, complementary and alternative medicine into the curriculum of South African medical schools. BMC Med Educ. 2014;14 (1):40. doi:10.1186/1472-6920-14-40

45. Falisse J-B, Masino S, Ngenzebuhoro R. Indigenous medicine and biomedical health care in fragile settings: insights from Burundi. Health Policy Plan. 2018;33(4):483-493. doi:10.1093/heapol/czy002

46. Aliyu UM, Awosan KJ, Oche MO, Taiwo AO, Jimoh AO, Okuofo EC. Prevalence and correlates of complementary and alternative medicine use among cancer patients in Usmanu Danfodiyo university teaching hospital, Sokoto, Nigeria. Niger J Clin Pract. 2017;20 (12):1576-1583. doi:10.4103/njcp.njcp_88_17

47. Aziato L, Clegg-Lamptey JNA. Breast cancer diagnosis and factors influencing treatment decisions in Ghana. Health Care Women Int. 2015;36(5):543-557. doi:10.1080/07399332.2014.911299

48. Muela SH, Mushi AK, Ribera JM. The paradox of the cost and affordability of traditional and government health services in Tanzania. Health Policy Plan. 2000;15(3):296-302. doi:10.1093/heapol/15.3.296

49. Leonard KL, Zivin JG. Outcome versus service based payments in health care: lessons from African traditional healers. Health Econ. 2005;14(6):575-593. doi:10.1002/hec.956

50. Nwankwo TO, AJah L, Ezeome IV, Umeh UA, Aniebue UU. Complementary and alternative medicine. Use and challenges among gynaecological cancer patients in Nigeria: experiences in a tertiary health institution - preliminary results. Eur J Gynaecol Oncol. 2019;40(1):101-105.

\section{Publish your work in this journal}

Cancer Management and Research is an international, peer-reviewed open access journal focusing on cancer research and the optimal use of preventative and integrated treatment interventions to achieve improved outcomes, enhanced survival and quality of life for the cancer patient.
The manuscript management system is completely online and includes a very quick and fair peer-review system, which is all easy to use. Visit http://www.dovepress.com/testimonials.php to read real quotes from published authors. 